\title{
Analytical Formalism for the Interaction of Two-Level Quantum Systems with Metal Nanoresonators
}

\author{
Jianji Yang, ${ }^{1}$ Mathias Perrin, ${ }^{2}$ and Philippe Lalanne ${ }^{1, *}$ \\ ${ }^{1}$ Laboratoire Photonique Numérique et Nanosciences, Institut d'Optique d'Aquitaine, \\ Université Bordeaux, CNRS, 33405 Talence, France \\ ${ }^{2}$ Laboratoire Ondes et Matière d'Aquitaine, Université Bordeaux, CNRS, 33405 Talence, France \\ (Received 25 November 2014; revised manuscript received 18 February 2015; published 17 April 2015) \\ Hybrid systems made of quantum emitters and plasmonic nanoresonators offer a unique platform \\ to implement artificial atoms with completely novel optical responses that are not available otherwise. \\ However, their theoretical analysis is difficult, and since many degrees of freedom have to be explored, \\ engineering their optical properties remains challenging. Here, we propose a new formalism that removes \\ most limitations encountered in previous analytical treatments and allows a flexible and efficient study \\ of complex nanoresonators with arbitrary shapes in an almost fully analytically way. The formalism \\ brings accurate closed-form expressions for the hybrid-system optical response and provides an intuitive \\ description based on the coupling between the quantum emitters and the resonance modes of the \\ nanoresonator. The ability to quickly predict light-scattering properties of hybrid systems paves the way to \\ a deep exploration of their fascinating properties and may enable rapid optimization of quantum plasmonic \\ metamaterials or quantum information devices.
}

DOI: 10.1103/PhysRevX.5.021008

\section{INTRODUCTION}

Localized surface-plasmon resonances of metallic nanoresonators have strong local field enhancements in deep-subwavelength mode volumes and offer unique opportunities to tailor light-matter interaction at the nanoscale [1,2]. Such opportunities become all the more remarkable as the near field of nanoresonators is dressed by two-level quantum emitters, such as atoms, molecules, or quantum dots. The resulting hybrid artificial system [3-9] indeed behaves very differently from its constituting elements and offers novel optical properties at the nanometer scale. For instance, under excitation by an external driving field, various Fano-like spectral responses could be observed, such as superabsorption in ultrasmall metallic particles [9], "cloaking" of the nanoresonator by the nearby quantum emitter [7,9], and unexpectedly broadband responses, much broader than the Purcell broadening, as will be shown. Hybrid systems can be seen as artificial excitons [8], whose optical properties (the resonance frequency, line shape, far-field radiation pattern, and near-field spatial and spectral distribution, etc.) can be engineered with great flexibility, and could become the

*Philippe.lalanne@institutoptique.fr

Published by the American Physical Society under the terms of the Creative Commons Attribution 3.0 License. Further distribution of this work must maintain attribution to the author(s) and the published article's title, journal citation, and DOI.
Subject Areas: Plasmonics, Quantum Information

building blocks for future generations of quantum plasmonic metamaterials or quantum information devices.

In theoretical works, to avoid quenching, two-level quantum emitters are assumed not to be located in the immediate vicinity of the metal so that the wave functions of the emitters and of the electron gas do not spatially overlap. The nanoresonator is then described by a dispersive permittivity, and in this limit, both the electromagnetic fields and the emitter degrees of freedom are treated as operators. The theoretical predictions of the optical properties generally rely on fully vectorial Green-tensor calculations. This approach necessitates tedious computations, which hide simple interpretations and need to be repeated whenever key parameters, such as the driving laser-field polarization, angle of incidence or frequency, and the quantum emitter polarization or location, are tuned for the analysis. Computational loads become prohibitive for hybrid systems made of multiple quantum objects [10-12] or complex metallic nanoparticles [3]. Clearly, the design of artificial plasmonic excitons requires new formalisms that restore physical intuition and analyticity.

A first classical approach relies on the quasistatic approximation and models the nanoresonator as an electric dipole $[4-6,13]$. Full analyticity and intuition are immediately achieved, but the approach is drastically limited to asymptotic cases. Analyticity is also achieved using Mie theory for textbook cases of spherical nanoresonator shapes [14]. For arbitrarily complex shapes, it was shown that a strong physical intuition can be restored [8] by an a posteriori fit of all possible coupling parameters involved in 
the relaxation dynamics, but the approach intrinsically relies on $a b$ initio fully vectorial computations of the system response.

Hereafter, we combine the recently developed quasinormal-mode (QNM) formalism [15,16] of plasmonic nanoresonators with the density-matrix formalism of two-level quantum systems $[9,17,18]$ and propose a totally new formalism that removes most limitations encountered in previous analytical approaches and allows us to handle complex nanoresonator shapes in an almost fully analytically way. One just needs to calculate the fundamental QNMs of the nanoresonator, but once the QNMs are known for a given nanoresonator shape, the hybrid-system response is obtained with closed-form expressions for any driving laser frequency, quantum emitter locations, polarization, etc. Computational loads are drastically reduced, and analyticity favors intuition and reveals general properties. The formalism is derived and tested for the canonical, albeit important, in practice, case of hybrids consisting of an arbitrary nanoresonator supporting one or several bright or dark modes dressed by a single two-level quantum emitter in the weak-coupling regime, but the formalism can be extended to other configurations with multiple quantum objects supporting collective effects or with three-level quantum systems. Other physical regimes, such as the nonlinear regime (with a strong driving field) [4] or strong emitter-plasmon coupling regimes [5], may also be explored in future works.

For our canonical geometry, we derive accurate and analytical expressions of the Fano line-shape spectral responses and evidence that analyticity offers a great flexibility for harnessing the optical properties of artificial plasmonic excitons. For instance, we reveal a new property, namely, the possibility to achieve spectral responses that are much broader than the spontaneous decay rate of the emitter; we deepen the general knowledge on the topic by proving that intriguing cloaking properties reported for some specific geometries in earlier works [7,9] are in reality observed with arbitrary geometries, the only requirement being that the emitter decay rate is boosted by the presence of the nanoresonator; we also predict and explain that the spectral response of the near-field electromagnetic intensity of geometries supporting higher-order (often dark) QNMs strongly varies in space, a fascinating property for developing new spectroscopic techniques at the nanoscale [19].

\section{QUASI-NORMAL-MODE AND DENSITY-MATRIX FORMALISMS}

Both formalisms are already documented in the literature, and it is not our intention to present them again hereafter. Rather, we focus on how they may be coupled to provide a fully analytical quantum treatment of hybrid plasmonic systems. As a reminder, the formalisms are first briefly presented and then the master equations leading to the coupling are derived in full for self-consistency. Technical details are appended in the methods.

The quantization of the electromagnetic radiation in dispersive and absorptive media has been initially developed by various authors [20,21]. These works were extended to inhomogeneous materials; see, e.g., Ref. [22] by writing a Hamiltonian for the quantized field in the vicinity of a metallic nanoparticle, the emitter, and the interaction. We adopt here the approach developed in Refs. $[9,17]$ and consider the quantum emitter as a twolevel system (TLS) with an electric dipolar transition. Under the rotating-wave approximation, and assuming a weak TLS-plasmon interaction, the dynamics of the expectation values is given by optical Bloch equations and the expectation value of the total field operator at the driving laser frequency $\omega_{L}$ reads as

$$
\langle\mathbf{E}(\mathbf{r})\rangle=\mathbf{E}_{L}(\mathbf{r})+\left\langle\mathbf{E}_{\mathrm{TLS}}(\mathbf{r})\right\rangle,
$$

where $\mathbf{E}_{L}(\mathbf{r})$, the coherent driving field, is treated as a classical quantity that represents the total field in the absence of the atom. $\left\langle\mathbf{E}_{\mathrm{TLS}}(\mathbf{r})\right\rangle$ is the expectation value of the field radiated by the TLS alone, which is treated as a point-dipole source placed at $\mathbf{r}_{0}$ in the vicinity of the plasmonic resonator. Note that an $\exp \left(i \omega_{L} t\right)$ dependence is assumed throughout the manuscript.

A rigorous quantum treatment $[9,23]$ shows that the self-action of the induced dipole reduces to a frequency shift (called a Lamb shift in a vacuum electromagnetic environment [24]), so that the Rabi frequency depends only on $\mathbf{E}_{L}\left(\mathbf{r}_{0}\right)$. Including the shift into the TLS resonance frequency, and neglecting the Rabi broadening, the expectation value for the induced-dipole moment reads as [9]

$$
\langle\mathbf{d}\rangle=\frac{-\boldsymbol{\mu}_{\mathrm{eg}} \Omega^{*}}{2 \delta_{L}-i \gamma},
$$

where $\boldsymbol{\mu}_{\mathrm{eg}}$ is the electric dipole moment of the electric dipolar transition between the ground and excited states. Hereafter, we assume a linearly polarized atomic transition and an ideal TLS whose damping is only driven by spontaneous radiative decay, but the formalism could be easily generalized to encompass saturation effects and nonradiative damping [9]. In Eq. (2), $\Omega=2 \boldsymbol{\mu}_{\mathrm{eg}}$. $\mathbf{E}_{L}^{*}\left(\mathbf{r}_{0}\right) / \hbar$ is the complex Rabi frequency, $\delta_{L}=$ $\omega_{L}-\omega_{\mathrm{TLS}}$ is the detuning between the laser driving frequency and the TLS resonance frequency $\omega_{\mathrm{TLS}}$, and $\gamma$ denotes the modified spontaneous decay rate of the excited state. The corresponding decay rate is derived from Fermi's golden rule [25] $\gamma=2 \operatorname{Im}\left(\boldsymbol{\mu}_{\mathrm{eg}}^{*} \mathbf{E}_{\mu}\left(\mathbf{r}_{0}\right)\right)$, where $\mathbf{E}_{\mu}\left(\mathbf{r}_{0}\right)$ is the field generated by the dipole source $\boldsymbol{\mu}_{\mathrm{eg}}$ at $\mathbf{r}_{0}$ in the presence of the nanoresonator. Details on the calculation of the TLS-dipole moment are given in Appendix A.

Quasinormal modes are the natural electromagnetic resonance modes of plasmonic resonators. They are 
eigensolutions of source-free Maxwell's equations with complex eigenfrequencies (due to the finite lifetime of the resonance). In the QNM formalism, the fields scattered by dissipative and dispersive resonant photonic or plasmonic nanoresonators are expressed as a superposition of modes. Even if the completeness of the modal expansion is not proven for dissipative plasmonic systems, it is shown that, close to resonance, accurate predictions can be obtained with expansions based only on a few dominant QNMs $[15,26]$. Hereafter, we adopt the approach developed in Refs. $[15,16]$, in which reciprocity arguments allow us to normalize the QNMs and to define an orthogonality relationship. The force of the QNM formalism resides in the fact that, once the QNMs are identified and properly normalized, one may analytically derive the electromagnetic response for various excitations and considerably lower the computational burden.

To combine both formalisms, we use a scattered-field formulation [16]. The total resonator response $\mathbf{E}\left(\mathbf{r}, \omega_{L}\right)$ at frequency $\omega_{L}, \mathbf{E}_{L}\left(\mathbf{r}, \omega_{L}\right)+\left\langle\mathbf{E}_{\mathrm{TLS}}(\mathbf{r})\right\rangle$ in the density-matrix approach, is written as a sum of the field $\mathbf{E}_{s}\left(\mathbf{r}, \omega_{L}\right)$ scattered by the nanoresonator and the background excitation $\mathbf{E}_{b}\left(\mathbf{r}, \omega_{L}\right)$ in the absence of the nanoresonator. By expanding $\mathbf{E}_{s}\left(\mathbf{r}, \omega_{L}\right)$ in the QNM basis, we have

$$
\mathbf{E}\left(\mathbf{r}, \omega_{L}\right) \approx \mathbf{E}_{b}\left(\mathbf{r}, \omega_{L}\right)+\sum_{m} c_{m}\left(\mathbf{E}_{b}, \omega_{L}\right) \tilde{\mathbf{E}}_{m}(\mathbf{r}),
$$

where $\left(\tilde{\mathbf{E}}_{m}, \tilde{\mathbf{H}}_{m}\right)$ and $\tilde{\omega}_{m}$ denote the field distribution of the $m$ th QNM and its complex eigenfrequency. The coupling coefficient $c_{m}\left(\mathbf{E}_{b}, \omega_{L}\right)$ is expressed as an overlap integral between the background field of the scattered-field formulation and the QNM

$$
\begin{aligned}
c_{m}\left(\mathbf{E}_{b}, \omega_{L}\right)= & -\frac{\omega_{L}}{\omega_{L}-\tilde{\omega}_{m}} \iiint \Delta \boldsymbol{\varepsilon}\left(\mathbf{r}, \omega_{L}\right) \mathbf{E}_{b}\left(\mathbf{r}, \omega_{L}\right) \\
& \cdot \tilde{\mathbf{E}}_{m}(\mathbf{r}) d^{3} \mathbf{r},
\end{aligned}
$$

where $\Delta \varepsilon(\mathbf{r}, \omega)$ denotes the difference between the nanoresonator permittivity and the background medium permittivity.
From Eqs. (1)-(4), in Appendix B, we derive the master equation of the present work

$$
\begin{aligned}
\langle\mathbf{E}(\mathbf{r})\rangle \approx & \mathbf{E}_{b}^{(L)}(\mathbf{r})+\mathbf{E}_{b}^{(\mathrm{TLS})}\left(\mathbf{r}, \tilde{\delta}_{L}\right) \\
& +\sum_{m} \beta_{m}\left(\omega_{\mathrm{TLS}}\right) \frac{2 \tilde{\delta}_{L}+i\left(f_{m}-1\right)}{2 \tilde{\delta}_{L}-i} \tilde{\mathbf{E}}_{m}(\mathbf{r}),
\end{aligned}
$$

which provides an analytical expression for the expectation value of the total field. The derivation relies on the sole approximation that plasmonic resonances are spectrally much broader than TLS resonances, an approximation largely justified even if the spontaneous decay of the TLS is boosted by a strong Purcell effect. In Eq. (5), $\tilde{\delta}_{L}=\delta_{L} / \gamma$ is the frequency detuning normalized by the Purcell-broadened TLS linewidth and

$$
f_{m}=-\alpha_{m}\left(\omega_{\mathrm{TLS}}\right) / \beta_{m}\left(\omega_{\mathrm{TLS}}\right)
$$

is a complex number that is called the modal Fano coefficient hereafter. It plays a key role in the following analysis; every QNM possesses its own Fano spectral response, with a line shape characterized by

$$
F_{m}(\omega)=\frac{2 \tilde{\delta}_{L}+i\left(f_{m}-1\right)}{2 \tilde{\delta}_{L}-i} .
$$

The master equation (5), which is illustrated in Fig. 1, constitutes the main useful result of the present formalism. Close to the TLS resonance frequency $\omega_{\mathrm{TLS}}$, the background terms in Eq. (5) are negligible for large Purcell effects (see Appendix C) and the vector-field response of the hybrid system is dominantly driven by a sum over the QNMs. Every expansion coefficient has a distinct modal Fano coefficient $f_{m}$. The latter accepts a very simple interpretation, as it compares the QNM excitation strengths at zero detuning $\alpha_{m}\left(\omega_{L}\right)$ and $\beta_{m}\left(\omega_{L}\right)$ due to the TLS and the driving laser (see Appendix B for details). As will be shown in Sec. IV, $f_{m}$ is a meaningful physical parameter that conveniently fully accounts for the broad variety of optical responses that can be achieved by slightly varying

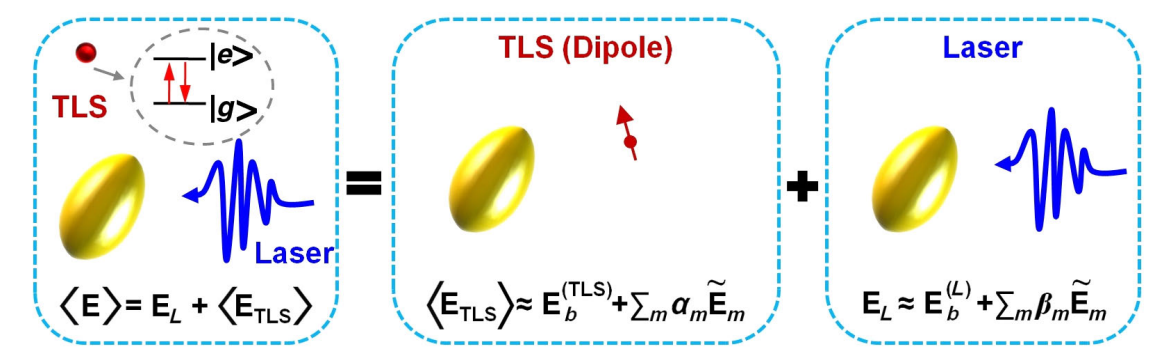

FIG. 1. Overview of the semianalytical modal formalism. The response of the hybrid system (left) to the laser driving field results from two contributions, the field radiated by the TLS and the field due to the driving excitation. The TLS and laser sources excite the QNMs of the nanoparticle, and we denote the respective expansion coefficients by $\alpha_{m}$ and $\beta_{m} . \mathbf{E}_{b}^{(\mathrm{TLS})}(\mathbf{r})$ and $\mathbf{E}_{b}^{(L)}(\mathbf{r})$ are, respectively, the background field associated with the TLS response and the external driving-field excitation, in the absence of the nanoresonator. 
the excitation conditions. For monomode resonators and for large Purcell factors, the spectral shape of the absorption is given by the Fano spectral response $\left|F_{m}\left(\omega_{L}\right)\right|^{2}$. The latter can be recast under a Fano shape $\left|F_{m}\left(\omega_{L}\right)\right|^{2}=$ $\left[\left(2 \tilde{\delta}_{L}+q_{+}\right)\left(2 \tilde{\delta}_{L}+q_{-}\right) /\left(2 \tilde{\delta}_{L}\right)^{2}+1\right]$, with $q_{ \pm}=-\operatorname{Im}(f) \pm$ $i[\operatorname{Re}(f)-1]$. For $\operatorname{Re}(f) \approx 1$ (which is always realized for high Purcell factors), $q_{+}=q_{-}=-\operatorname{Im}(f)$, and one exactly recovers the formula introduced by Ugo Fano himself [27]. The analytical formalism clearly evidences that the pure Fano line shape results from the coupling of the laser and the TLS to the resonance mode of the resonator, and not from an intricate interference with the background fields.

\section{IMPLEMENTATION, EFFECTIVENESS, AND NUMERICAL TESTS}

In practice, to calculate Eq. (5), one first needs to compute the QNMs. This computation can be performed with the general method described in Ref. [16]. Hereafter, we use an open-source code [28]. The calculation per QNM, which requires 4-5 iterations at complex frequencies starting from an initial guess, generally lasts less than 5 min and a low-speed workstation. Once the QNMs are known, then $\mathbf{E}_{L}(\mathbf{r})$, the Rabi frequency, and the Purcell factor are obtained analytically, as explained in Appendix A. The expectation value $\langle\mathbf{d}\rangle$ of the induced dipole is then known, and the total response is calculated from Eq. (5). Supplemental technical details are given in Appendixes B and C.

To test the validity of the semianalytical formalism, we consider a canonical arrangement, consisting of a small 5 -nm-diameter gold sphere embedded in a glass background $(n=1.5)$ and coupled to a TLS with a radial dipole moment. The responses of such systems have already been studied intensively [4-6,9] by resorting to either Mie theory or a dipolar approximation. To compare with data available from Ref. [9], the TLS is assumed to be placed $18 \mathrm{~nm}$ above the sphere [see Fig. 2(b)] with a resonance wavelength $\lambda_{\mathrm{TLS}}=545 \mathrm{~nm}$ (Purcell factor of approximately 2.2) and a natural linewidth $\gamma_{0}=75 \mathrm{MHz}$ in vacuum. The same TLS will be considered throughout the article. The gold sphere exhibits a dipolar plasmonic resonance around $545 \mathrm{~nm}$, with a complex eigenwavelength $\lambda_{e}=548-22 i \mathrm{~nm}$.

We analyze the response of the hybrid system to a linearly polarized plane wave, whose electric field is collinear to the TLS-dipole moment. There, we assume that a single dipolar QNM contributes to the field expansion and compute the value of the modal Fano coefficient $f=1+33 i$ from Eqs. (4) and (10). Afterwards, both the scattering (blue curve) and absorption (red curve) cross sections, respectively denoted by $\sigma_{\text {sca }}$ and $\sigma_{\text {abs }}$, are easily obtained (see Appendix C). Figure 2(a) compares the predictions of our QNM formalism with "exact" computational results obtained with Mie theory [9]. Quantitative agreement is reached for the nearly symmetric line shape of
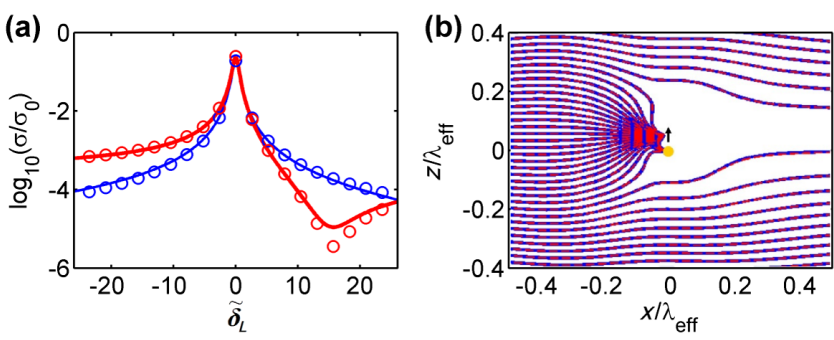

FIG. 2. Test of the accuracy of the semiclassical modal formalism for a TLS weakly coupled to a nanosphere. The arrangement consists in a gold nanosphere (5-nm diameter) at a distance of $18 \mathrm{~nm}$ from a TLS with resonance wavelength $\lambda_{\mathrm{TLS}}=545 \mathrm{~nm}$, oriented radially [9] in a glass background $(n=1.5)$. (a) Scattering (blue) and absorption (red) cross sections, normalized to the extinction cross section $\sigma_{0}=$ $\left(3 \lambda_{\text {TLS }}^{2} / 2 \pi n^{2}\right)$ of a bare TLS in glass, as a function the laser detuning. Solid lines and circles refer, respectively, to the present analytical formalism and Mie theory. (b) At zero detuning, Poynting-flux lines calculated with Mie theory (solid blue lines) and the present formalism (dashed red lines) are almost superimposed. The driving plane wave flows from left to right with a vertical polarization and $\lambda_{\text {eff }}=\lambda_{L} / n$. Note an error (factor 10) in the scale of the horizontal axis in Fig. 1(b) in Ref. [9]: Actually, the spectral width of the optical response is much larger than the Purcell broadening.

$\sigma_{\text {sca }}$ and the asymmetric Fano line shape of $\sigma_{\text {abs }}$ with a large enhancement at zero detuning and a strong reduction at $\tilde{\delta}_{L}=16.5$. The difference in line shapes of both cross sections is due to the fact that $\mathbf{E}_{b}^{(\mathrm{TLS})}\left(\mathbf{r}, \tilde{\delta}_{L}\right)$ dominates over the QNM field outside the nanoparticle for small Purcell factors. This fact adds up a Lorentzian response that results in a symmetric line shape for $\sigma_{\text {sca. }}$. However, $\sigma_{\text {abs }}$ that depends on the field inside the nanoparticle has a typical Fano line shape.

The capability and accuracy of the QNM formalism are further evidenced in Fig. 2(b), where the flux lines calculated at zero detuning with the QNM formalism are shown to be almost superimposed with the ones provided by Mie theory. For the sake of compactness, we present an accuracy test just for a single particular canonical arrangement; however, we have verified that the QNM formalism provides accurate predictions for many other arrangements, including all those studied in the following sections.

Equation (5) offers the possibility to analytically calculate the spectral response of the hybrid system for any driving laser frequency, shape, or polarization or quantum emitter locations and polarizations. This formalism removes most limitations encountered in previous approaches and allows us to handle complex resonator shapes in an almost fully analytically way. In return, the approach is approximate. For instance, it is likely not to be able to account for quenching, which may require us to calculate very high-order QNMs. Another drawback is that it is difficult to estimate the error made by retaining only a few QNMs. This query would require a comparison with 
fully vectorial data, but note that this would require only a few comparisons, since one may reasonably expect that the accuracy of the method does not change much by varying the geometrical parameters. This fact has been noticed for some particular structures, such as cylinders. Finally, note that in experiments, the exact shape of the resonator, location, and/or orientation of the emitter may be known only approximately and that highly accurate predictions are not critical to well understand the physics of the problem at hand (symmetries, quenching, number of modes, etc.) and to interpret the experimental data. In fact, the present method may help. For example, by introducing a statistical variation of geometry, index of refraction, etc., and by computing a distribution of modes, one may obtain a statistical distribution of extinction or absorption spectra, directly related to experimental uncertainties, in a rapid way.

\section{FANO LINE SHAPES OF MONOMODE HYBRIDS}

In this section, we consider hybrids that are dominantly driven by a single QNM. We will see that these monomode hybrids exhibit a large richness of optical responses that are easily tuned by slightly changing the hybrid geometry and are elegantly predicted by the analytical model with a single complex parameter, the Fano coefficient.

As is well known, the optical response of monomode hybrids in the general case presents a nonsymmetric line shape with a local maximum (peak) and a local minimum (dip), and the overall shape largely varies by tuning some key internal parameters of the hybrid. This effect is due to the interference that takes place between a continuum (the broad plasmonic resonance) and the sharp TLS resonance.
As illustrated in Fig. 3, the great variety of response patterns is well described under a monomode approximation with a single generic Fano function $\left|F\left(\tilde{\delta}_{L}\right)\right|$, in which the modal Fano coefficient $f$ varies as the internal parameters are tuned. We emphasize that the different line shapes in Fig. 3 are all obtained analytically with a single Fano function whose Fano coefficient is known analytically, and that we have checked that the Fano function accurately predicts the absorption spectra of each of the cases taken into consideration.

With Fig. 3(a), we consider typical responses characterized by large values of $\operatorname{Im}(f)$, for which the Fano responses exhibit marked dips and peaks; the dip strikingly goes to almost 0 at $\tilde{\delta}_{L}=\operatorname{Im}(f) / 2$, and the peak reaches a large value $\sqrt{1+\operatorname{Im}(f)^{2}}$ at $\tilde{\delta}_{L} \approx 0$. In contrast, with Figs. 3(b) and 3(c), we consider typical responses with small values of $\operatorname{Im}(f)$; the peak and dip collide, and only a single nearly symmetric dip, approximately centered around $\tilde{\delta}_{L}=0$, remains present in the spectrum.

The contrasted response of Fig. 3(a) admits a simple physical interpretation by considering that the TLS acts as an antenna with a scattering cross section that is much larger than that of the nanoresonator, i.e., $\left|\alpha\left(\omega_{\mathrm{TLS}}\right)\right| \gg|\beta|$. Surprisingly, we find that the frequency interval between the peak and the dip is not bounded by the spontaneous decay rate $\gamma$ of the excited level as one may intuitively expect; actually, it may be more than 10 times larger than the Purcell-broadened linewidth. We also note that the frequency interval rapidly varies with the separation distance $\Delta$, an important property that may be helpful for experimental characterization and that is analytically predicted with the modal formalism.
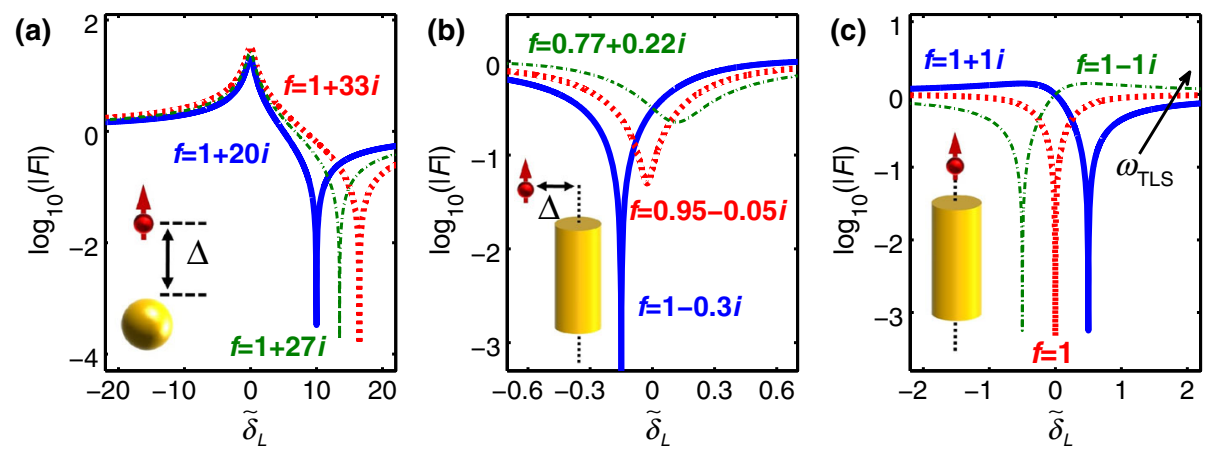

FIG. 3. Diversity of the Fano responses for hybrid systems made of single-mode metallic nanoresonators. (a),(b) Fano line shapes obtained by varying the TLS position. (a) The hybrid-system configuration studied in Fig. 2 with three different emitter-sphere separations: $\Delta=12.5 \mathrm{~nm}$ (solid blue line), $18 \mathrm{~nm}$ (dashed red line), and $23.5 \mathrm{~nm}$ (thin dot-dashed green line). The corresponding Purcell factors are 9.2, 2.2, and 1.3. In (b), the TLS $\left(\lambda_{\mathrm{TLS}}=900 \mathrm{~nm}\right)$ is located $10 \mathrm{~nm}$ above a gold nanorod (radius $15 \mathrm{~nm}$, length $100 \mathrm{~nm}$, and dipolar plasmonic resonance at eigenwavelength $\lambda_{e}=918-47 i \mathrm{~nm}$ ) and is shifted toward the driving field by a distance $\Delta=25 \mathrm{~nm}$ (solid blue line), $50 \mathrm{~nm}$ (dashed red line), and $75 \mathrm{~nm}$ (thin dot-dashed green line). The associated Purcell factors are 55 , 2 , and 0.7. (c) Fano line shapes obtained for large Purcell factors and for a TLS placed on axis 10 nm above the nanorod. The TLS resonance is tuned from $\lambda_{\mathrm{TLS}}=960 \mathrm{~nm}$ (solid blue line) to $917 \mathrm{~nm}$ (dashed red line, at the plasmonic resonance), and $840 \mathrm{~nm}$ (thin dotdashed green line). The corresponding Purcell factors are 295, 412, and 70, respectively. For the simulation, a gold relative permittivity given by a Drude model $\varepsilon_{m}=1-\omega_{p}^{2} /\left(\omega^{2}-i \omega \Gamma\right)$ is adopted with $\omega_{p}=1.26 \times 10^{16} \mathrm{~s}^{-1}$ and $\Gamma=1.41 \times 10^{14} \mathrm{~s}^{-1}$. In all cases, the driving plane wave propagates from left to right with a vertical polarization (same as the one of the TLS). 
A further important case, in practice, corresponds to hybrid systems operating with large Purcell factors. Generally, the position $\mathbf{r}_{0}$ at which a TLS experiences large decay accelerations is always associated with a strong field enhancement under far-field laser excitation, provided that the TLS and the laser indeed have the same polarization. Thus, assuming that $\mathbf{E}_{L}\left(\mathbf{r}_{0}\right) \approx \beta \tilde{\mathbf{E}}\left(\mathbf{r}_{0}\right)$, from Eq. (6), it is found that the implementation of large Purcell factors requires $\operatorname{Re}(f) \approx 1$, as is consistently observed in Figs. 3(b) (blue curve only) and 3(c), as well as with other results (not shown) obtained for a spherical dimer [29]. Although the relation $\operatorname{Re}(f) \approx 1$ largely restricts the variety of possible response patterns, $\operatorname{Im}(f)$ is not imposed and, by tuning the TLS resonant frequencies within the plasmonic resonance bandwidth, the Purcell factor remains large and the spectral responses may still be significantly tuned, from a symmetric line shape with a dip at zero detuning for $\operatorname{Im}(f)=0$ to asymmetric line shapes with dip frequencies that may be either red- or blueshifted; see Fig. 3(c). Note that for large emission rates, the Fano function $F$ determines both the scattering and absorption cross sections. Thus, deep dips of $|F|$, such as those of Fig. 3(c), result in very weak extinction cross sections, which make the nanoresonator hardly visible (or cloaked) for the far field.

\section{MULTIMODE ARRANGEMENTS}

So far, we have been considering the interaction of quantum emitters with metal nanoparticles supporting a single resonance. One of the great values of the present method is its capability to handle multiresonance cases in a very intuitive manner, since the impact of each resonance is naturally and separately considered in the master equation. Otherwise, intuition would be considerably weakened and computational loads would be drastically enhanced.
From Eq. (5), the expectation value of each field component $X=E_{x}, E_{y}, E_{z}, H_{x}, H_{y}$, or $H_{z}$ may be conveniently written as

$$
\begin{aligned}
\langle X(\mathbf{r})\rangle \approx & X_{b}^{(L)}(\mathbf{r})+X_{b}^{(\mathrm{TLS})}(\mathbf{r}) \\
& +\sum_{m} \beta_{m}\left(\omega_{\mathrm{TLS}}\right) \tilde{X}_{m}(\mathbf{r}) \frac{2 \tilde{\delta}_{L}+i\left[f_{X}(\mathbf{r})-1\right]}{2 \tilde{\delta}_{L}-i},
\end{aligned}
$$

if one introduces a generalized Fano coefficient $f_{X}(\mathbf{r})=$ $\sum_{m} \beta_{m}\left(\omega_{\mathrm{TLS}}\right) \tilde{X}_{m}(\mathbf{r}) f_{m} / \sum_{m} \beta_{m}\left(\omega_{\mathrm{TLS}}\right) \tilde{X}_{m}(\mathbf{r})$, defined as the weighted mean value of the individual modal Fano coefficients. If the $f_{m}$ 's are different, $f_{X}(\mathbf{r})$ depends on the space coordinate. Thus, in sharp contrast to single-mode cases, hybrid systems made of a multimode plasmonic resonator offer spectral line-shape responses that considerably vary in the near-field zone of the nanoresonator. In particular, even if the far-field response may remain driven by a predominant single radiative QNM, high-order modes such as electric quadrupoles that weakly radiate could efficiently couple to the TLS and offer a myriad of contrasted spectral responses in the near field, a fascinating property that could be exploited to develop new imaging and spectroscopic techniques at the nanoscale [19]. The TLS acts as an auxiliary resonant structure that effectively transfers the far-field radiation to high-order dark modes of the resonator, via the resonant excitation of the lowest-order bright mode. Based on this mode conversion, scattered optical near fields can be well manipulated by utilizing the interference between different resonator modes.

To exemplify our purpose, we consider a heterodimer composed of two gold nanorods of different sizes (radii $R_{1}=20 \mathrm{~nm}$ and $R_{2}=85 \mathrm{~nm}$, lengths $L_{1}=80 \mathrm{~nm}$ and $L_{2}=145 \mathrm{~nm}$, gap size $45 \mathrm{~nm}$ ), of which the optical response in the spectral range 700-1200 $\mathrm{nm}$ is mainly
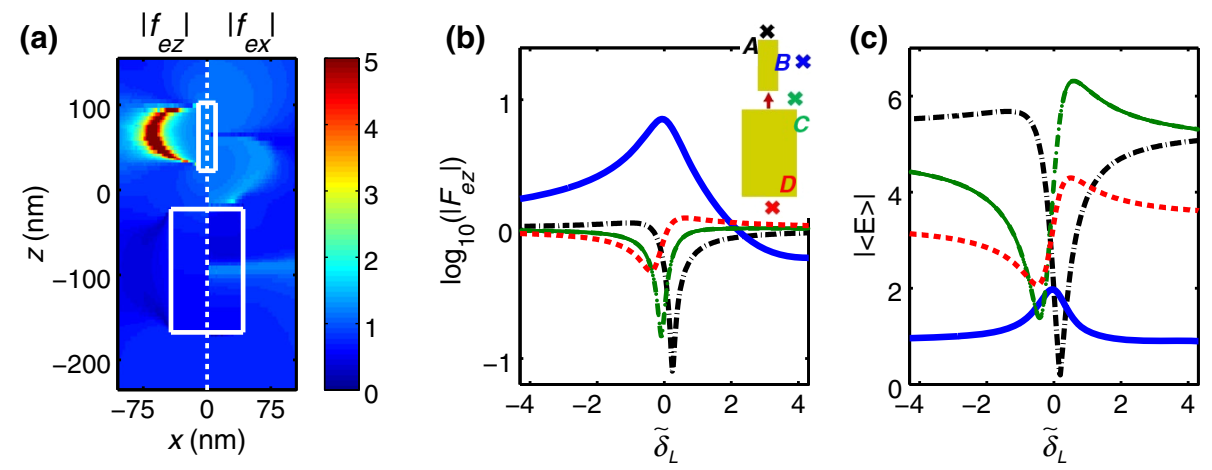

FIG. 4. Spectral response in the near-field zone of a multimode artificial hybrid system. (a) Complex Fano coefficients $\left|f_{e z}\right|$ (left side) and $\left|f_{e x}\right|$ (right side) in the $x-z$ midplane of a nanorod heterodimer. (b) Modulus of the Fano function $F_{e z}$ at locations $A$ (black line), $B$ (blue line), $C$ (green line), and $D$ (red line). The corresponding $f_{e z}$ values are $0.91+0.49 i, 5.24+5.61 i, 0.85-0.16 i$, and $0.42-0.61 i$. The near-field location coordinates are $A(0 \mathrm{~nm}, 130 \mathrm{~nm}), B(60 \mathrm{~nm}, 60 \mathrm{~nm}), C(35 \mathrm{~nm}, 0 \mathrm{~nm})$, and $D(0 \mathrm{~nm},-180 \mathrm{~nm})$. (c) Spectra of the modulus of the local field expectation value $\langle\mathbf{E}\rangle$. The inset in (b) sketches the probed locations. The external driving field propagates from left to right with a vertical polarization. [Note that the model predictions in (c) are accurately reproduced with fully vectorial Green-tensor calculations.] 
driven by two QNMs with eigenwavelengths $\lambda_{e 1}=933-$ $77 i \mathrm{~nm}$ and $\lambda_{e 2}=958-108 i \mathrm{~nm}$ [15]. In the center of the dimer gap, a $z$-polarized TLS is placed, with a transition wavelength $\lambda_{\mathrm{TLS}}=990 \mathrm{~nm}$, at which the Purcell factor reaches a maximum value of approximately 155 .

Figure 4(a) shows the absolute value of the Fano coefficient $f_{e z}(\mathbf{r})$ and $f_{e x}(\mathbf{r})$ of the $z$ and $x$ components of the electric field in the $x-z$ midplane. The spatial distributions are highly nonuniform and strongly differ from one component to the other, especially around the upper arm. As a consequence, for a different field component $X$, distinctive Fano line shapes $F_{X}(\mathbf{r})=\left(2 \tilde{\delta}_{L}+\right.$ $\left.i\left[f_{X}(\mathbf{r})-1\right]\right) /\left(2 \tilde{\delta}_{L}-i\right)$ are obtained as $\mathbf{r}$ is varied. For the dominant field component $\mathbf{E}_{z}$, the Fano line shapes $F_{e z}$ are plotted in Fig. 4(b) for sampling locations labeled by $A$, $B, C$, and $D$, in the near-field region of the dimer. Line shapes at $A, C$, and $D$ are similar to those obtained for the monomode case in Fig. 3, but the one at $B$ is remarkably different because of its unusual Fano coefficient value (see the caption). In Fig. 4(c), we show the corresponding line shapes of the local field expectation value $|\langle\mathbf{E}\rangle|$, for which $\left|\left\langle\mathbf{E}_{z}\right\rangle\right| \approx|\langle\mathbf{E}\rangle|$ is dominant. Clearly, the local field spectra drastically vary at the nanometer scale, due to the interference between several modes, with distinct and spatially dependent modal Fano coefficients.

\section{CONCLUSION}

In summary, we have derived an analytical formalism based on quasi-normal-mode expansions for the study of the optical properties of plasmonic systems interacting with quantum objects (e.g., molecules, atoms, or quantum dots). In agreement with previous works [4-9], we show that the spectral response exhibits Fano resonances resulting from the interaction between the quantum objects and the plasmon resonance. Importantly and uniquely to our knowledge, the formalism allows the derivation of accurate closed-form expressions for the Fano factor, which can be directly calculated without fit even for complex geometries. The flexibility added by the analyticity is amazingly comfortable, in practice, since it favors an in-depth understanding and allows a very efficient computation of the optical responses when some key physical parameters are modified. As a straightforward application, let us mention the possibility to rapidly evaluate ensemble-averaged responses over a distribution of TLS orientations, positions, and resonance frequencies. This formalism could help the interpretation of experimental measurement on dilute samples, in which the artificial plasmonic excitons are neither identical nor identically oriented. With the present modal approach, analyticity can be preserved for more complicated systems, such as amplifying systems, by modifying the optical Bloch equations to model the gain with a TLS driven out of thermodynamic equilibrium by a pump field [30]. The formalism can also be directly extended toward hybrids formed by a nanoresonator dressed by several TLSs to study superradiance or spasing. Our recent efforts in that direction are very encouraging.

Generally speaking, we believe that theoretical formalisms, which explicitly rely on the natural resonances of the plasmonic nanoresonator, represent an enabling approach for the description of plasmon-exciton interactions in complex systems.

\section{ACKNOWLEDGEMENTS}

The authors thank C. Sauvan and J. P. Hugonin for fruitful discussions and help. Part of the study was carried out with financial support from "the Investments for the future" Programme IdEx Bordeaux-LAPHIA (ANR-10IDEX-03-02).

\section{APPENDIX A: CALCULATION OF RABI FREQUENCY $\Omega$ AND DECAY RATE $\gamma$}

In order to compute the Rabi frequency $\Omega=2 \boldsymbol{\mu}_{\mathrm{eg}}$. $\mathbf{E}_{L}{ }^{*}\left(\mathbf{r}_{0}\right) / \hbar$ and the decay rate $\gamma=2 \operatorname{Im}\left(\boldsymbol{\mu}_{\mathrm{eg}}^{*} \mathbf{E}_{\mu}\left(\mathbf{r}_{0}\right)\right) / \hbar$, one needs to calculate $\mathbf{E}_{L}\left(\mathbf{r}_{0}\right)$ and $\mathbf{E}_{\mu}\left(\mathbf{r}_{0}\right)$. For that purpose, we expand $\mathbf{E}_{L}\left(\mathbf{r}_{0}\right)$ and $\mathbf{E}_{\mu}(\mathbf{r})$ in the QNM basis according to Eq. (3) and calculate the expansion coefficients with Eq. (4). Note that, according to Eq. (2), the transitiondipole moment $\boldsymbol{\mu}_{\mathrm{eg}}$ and the expectation value of the induced-dipole moment $\langle\mathbf{d}\rangle$ are parallel. Thus, the field $\left\langle\mathbf{E}_{\mathrm{TLS}}(\mathbf{r})\right\rangle$ emitted by $\langle\mathbf{d}\rangle$ is simply proportional to the field $\mathbf{E}_{\mu}(\mathbf{r})$ emitted by $\boldsymbol{\mu}_{\mathrm{eg}}$ and can be obtained easily from $\mathbf{E}_{\mu}(\mathbf{r}),\left\langle\mathbf{E}_{\mathrm{TLS}}(\mathbf{r})\right\rangle=\left(-\Omega^{*} / 2 \delta_{L}-i \gamma\right) \mathbf{E}_{\mu}(\mathbf{r})$.

\section{APPENDIX B: DERIVATION OF THE MASTER EQUATION}

The derivation of Eq. (5) starts by applying Eq. (3) to each term of the right-hand side of Eq. (1). Dropping the dependency on $\omega_{L}$, we obtain

$$
\begin{array}{r}
\mathbf{E}_{L}(\mathbf{r}) \approx \mathbf{E}_{b}^{(L)}(\mathbf{r})+\sum_{m} \beta_{m} \tilde{\mathbf{E}}_{m}(\mathbf{r}), \\
\left\langle\mathbf{E}_{\mathrm{TLS}}(\mathbf{r})\right\rangle \approx \mathbf{E}_{b}^{(\mathrm{TLS})}(\mathbf{r})+\sum_{m} \alpha_{m} \tilde{\mathbf{E}}_{m}(\mathbf{r}),
\end{array}
$$

where $\mathbf{E}_{b}^{(L)}(\mathbf{r})$ and $\mathbf{E}_{b}^{(\mathrm{TLS})}(\mathbf{r})$ are, respectively, the background fields (the illumination fields in the absence of a nanoresonator) associated with the laser excitation and with the radiation by a TLS of electric dipole moment $\langle\mathbf{d}\rangle$. The coupling coefficients $\alpha_{m}\left(\omega_{L}\right)=c_{m}\left(\mathbf{E}_{b}^{(\mathrm{TLS})}, \omega_{L}\right)$ and $\beta_{m}\left(\omega_{L}\right)=c_{m}\left(\mathbf{E}_{b}^{(L)}, \omega_{L}\right)$ are known analytically. From Eqs. (B1) and (B2), one obtains the modal representation of the expectation value of the total field (see Fig. 1) 


$$
\langle\mathbf{E}(\mathbf{r})\rangle \approx \mathbf{E}_{b}^{(L)}(\mathbf{r})+\mathbf{E}_{b}^{(\mathrm{TLS})}(\mathbf{r})+\sum_{m}\left[\alpha_{m}+\beta_{m}\right] \tilde{\mathbf{E}}_{m}(\mathbf{r}) .
$$

Plasmonic resonances of metal nanoparticles have low quality factors $(Q<100)$ limited by Ohmic and radiation losses and are spectrally much broader than TLS resonances. Thus, the laser expansion coefficient $\beta_{m}$ can be considered as constant over the spectral width of the TLS resonance, i.e., $\beta_{m}(\omega) \approx \beta_{m}\left(\omega_{\text {TLS }}\right)$. In sharp contrast, the TLS expansion coefficient $\alpha_{m}$ rapidly varies around the TLS resonance frequency. Thus, from Eq. (2), one may write

$$
\alpha_{m}\left(\omega_{L}\right)=\frac{-i \gamma}{2\left(\omega_{L}-\omega_{\mathrm{TLS}}\right)-i \gamma} \alpha_{m}\left(\omega_{\mathrm{TLS}}\right),
$$

with $\alpha_{m}\left(\omega_{\text {TLS }}\right)$ the expansion coefficient of the $m$ th mode at zero detuning $\omega=\omega_{\mathrm{TLS}}$, for which the dipole moment of the TLS is $-i \mu_{\mathrm{eg}} \Omega^{*} / \gamma$. By introducing the frequency detuning normalized by the Purcell-broadened TLS linewidth $\tilde{\delta}_{L}=\delta_{L} / \gamma$, the expectation value of the total field of Eq. (5) is obtained.

\section{APPENDIX C: CALCULATION OF $\sigma_{\mathrm{abs}}, \sigma_{\mathrm{ext}}, \mathrm{AND} \sigma_{\mathrm{sca}}$}

The response of the hybrid system can be quantified by the absorption cross section $\sigma_{\mathrm{abs}}$, extinction cross section $\sigma_{\text {ext }}$, and scattering cross section $\sigma_{\text {sca }}=\sigma_{\text {ext }}-\sigma_{\text {abs }}$. It should be first stated that the entire hybrid system is a passive system, excited only by the driving laser beam, so that all the cross sections are defined with respect to the incident power of the driving field.

We calculate the absorption cross section $\sigma_{\text {abs }}$ with the following formula:

$$
\sigma_{\mathrm{abs}}=-\frac{\omega}{2 \mathcal{S}_{0}} \iiint_{V} \operatorname{Im}[\varepsilon(\mathbf{r}, \omega)]|\langle\mathbf{E}(\mathbf{r})\rangle|^{2} d^{3} \mathbf{r},
$$

which represents a volume integral running only over the metallic nanoparticle, as we consider only an ideal TLS here (no absorption). $\mathcal{S}_{0}$ denotes the time-averaged Poynting vector of the incident plane wave, and $\langle\mathbf{E}(\mathbf{r})\rangle$ is the expectation value of the total field; see Eq. (5). We empirically remark that the background TLS field $\mathbf{E}_{b}^{(\mathrm{TLS})}$ of the dipole source can be neglected inside the resonator and the background laser field $\mathbf{E}_{b}^{(L)}$ is relatively weak when the QNMs are resonantly excited (close to the plasmonic resonance); thus, inside the resonator, we approximately have $\langle\mathbf{E}(\mathbf{r})\rangle \approx \sum_{m}\left[\alpha_{m}+\beta_{m}\right] \tilde{\mathbf{E}}_{m}(\mathbf{r})$. Therefore, when a single QNM is excited, the spectral line shape of $\sigma_{\mathrm{abs}}$ is determined by $|\alpha+\beta|^{2}$ or by the square of the modulus of the Fano function of Eq. (7).
For a good accuracy, we calculate the extinction cross section $\sigma_{\text {ext }}$ of the hybrid system as

$$
\begin{aligned}
\sigma_{\mathrm{ext}}= & \sigma_{\mathrm{ext}}^{\mathrm{TLS}}+\sigma_{\mathrm{ext}}^{\mathrm{NR}} \\
= & -\frac{\omega}{2 \mathcal{S}_{0}} \operatorname{Im}\left[\mathbf{d} \cdot \mathbf{E}_{b}^{(L) *}\left(\mathbf{r}_{0}\right)\right] \\
& -\frac{\omega}{2 \mathcal{S}_{0}} \iiint_{V} \operatorname{Im}\left[\Delta \varepsilon(\mathbf{r}, \omega)\langle\mathbf{E}(\mathbf{r})\rangle \cdot \mathbf{E}_{b}^{(L) *}(\mathbf{r})\right] d^{3} \mathbf{r} .
\end{aligned}
$$

Equation (C2) does not correspond to the usual expression for the extinction cross section [31] but allows us to distinguish the TLS and resonator contributions $\left(\sigma_{\mathrm{ext}}^{\mathrm{TLS}}\right)$ and $\left(\sigma_{\mathrm{ext}}^{\mathrm{NR}}\right)$.

Finally, the scattering cross section is calculated as $\sigma_{\mathrm{sca}}=\sigma_{\mathrm{ext}}-\sigma_{\mathrm{abs}}$.

[1] Y. Liu and X. Zhang, Metamaterials: A New Frontier of Science, and Technology, Chem. Soc. Rev. 40, 2494 (2011).

[2] P. Berini and I. De Leon, Surface Plasmon-Polariton Amplifiers, and Lasers, Nat. Photonics 6, 16 (2011).

[3] A. O. Govorov, G. W. Bryant, W. Zhang, T. Skeini, J. Lee, N. A. Kotov, J. M. Slocik, and R. R. Naik, Exciton-Plasmon Interaction, and Hybrid Excitons in Semiconductor-Metal Nanoparticle Assemblies, Nano Lett. 6, 984 (2006).

[4] W. Zhang, A. O. Govorov, and G. W. Bryant, SemiconductorMetal Nanoparticle Molecules: Hybrid Excitons, and the Nonlinear Fano Effect, Phys. Rev. Lett. 97, 146804 (2006).

[5] R. D. Artuso and G. W. Bryant, Optical Response of Strongly Coupled Quantum Dot-Metal Nanoparticle Systems: Double Peaked Fano Structure, and Bistability, Nano Lett. 8, 2106 (2008).

[6] A. Ridolfo, O. Di Stefano, N. Fina, R. Saija, and S. Savasta, Quantum Plasmonics with Quantum Dot-Metal Nanoparticle Molecules: Influence of the Fano Effect on Photon Statistics, Phys. Rev. Lett. 105, 263601 (2010).

[7] X. Wu, S. K. Gray, and M. Pelton, Quantum Dot Induced Transparency in a Nanoscale Plasmonic Resonator, Opt. Express 18, 23633 (2010).

[8] A. Manjavacas, F. J. Garcia de Abajo, and P. Nordlander, Quantum Plexcitonics: Strongly Interacting Plasmons, and Excitons, Nano Lett. 11, 2318 (2011).

[9] X. Chen, V. Sandoghdar, and M. Agio, Coherent Interaction of Light with a Metallic Structure Coupled to a Single Quantum Emitter: From Superabsorption to Cloaking, Phys. Rev. Lett. 110, 153605 (2013).

[10] M. Creasey, J.-H. Lee, Z. Wang, G. J. Salamo, and X. Li, Self-Assembled InGaAs Quantum Dot Clusters with Controlled Spatial, and Spectral Properties, Nano Lett. 12, 5169 (2012).

[11] R. Schreiber, J. Do, E.-M. Roller, T. Zhang, V. J. Schüller, P. C. Nickels, J. Feldmann, and T. Liedl, Hierarchical Assembly of Metal Nanoparticles, Quantum Dots, and 
Organic Dyes Using DNA Origami Scaffolds, Nat. Nanotechnol. 9, 74 (2014).

[12] K. D. B. Higgins, S. C. Benjamin, T. M. Stace, G. J. Milburn, B. W. Lovett, and E. M. Gauger, Superabsorption of Light via Quantum Engineering, Nat. Commun. 5, 4705 (2014).

[13] D. E. Gómez, H. Giessen, and T. J. Davis, Semiclassical Plexcitonics: Simple Approach for Designing Plexcitonic Nanostructures, J. Phys. Chem. C 118, 23963 (2014).

[14] V. N. Pustovit and T. V. Shahbazyan, Cooperative Emission of Light by an Ensemble of Dipoles Near a Metal Nanoparticle: The Plasmonic Dicke Effect, Phys. Rev. Lett. 102, 077401 (2009).

[15] C. Sauvan, J. P. Hugonin, I. S. Maksymov, and P. Lalanne, Theory of the Spontaneous Optical Emission of Nanosize Photonic, and Plasmonic Resonators, Phys. Rev. Lett. 110, 237401 (2013).

[16] Q. Bai, M. Perrin, C. Sauvan, J. P. Hugonin, and P. Lalanne, Efficient, and Intuitive Method for the Analysis of Light Scattering by a Resonant Nanostructure, Opt. Express 21, 27371 (2013).

[17] C. Girard, O. J. Martin, G. Leveque, G. C. des Francs, and A. Dereux, Generalized Bloch Equations for Optical Interactions in Confined Geometries, Chem. Phys. Lett. 404, 44 (2005).

[18] S. Severini, A. Settimi, C. Sibilia, M. Bertolotti, A. Napoli, and A. Messina, Second Quantization, and Atomic Spontaneous Emission inside One-Dimensional Photonic Crystals via a Quasinormal-Modes Approach, Phys. Rev. E 70, 056614 (2004).

[19] D. Brinks, M. Castro-Lopez, R. Hildner, and N. F. van Hulst, Plasmonic Antennas as Design Elements for Coherent
Ultrafast Nanophotonics, Proc. Natl. Acad. Sci. U.S.A. 110, 18386 (2013).

[20] B. Huttner and S. M. Barnett, Quantization of the Electromagnetic Field in Dielectrics, Phys. Rev. A 46, 4306 (1992).

[21] R. Matloob, R. Loudon, S. M. Barnett, and J. Jeffers, Electromagnetic Field Quantization in Absorbing Dielectrics, Phys. Rev. A 52, 4823 (1995).

[22] L. Knöll, S. Scheel, and D.-G. Welsch, in Coherence and Statistics of Photons and Atoms, edited by J. Perina (Wiley, New York, 2001).

[23] D. Martín-Cano, H. R. Haakh, K. Murr, and M. Agio, Large Suppression of Quantum Fluctuations of Light from a Single Emitter by an Optical Nanostructure, Phys. Rev. Lett. 113, 263605 (2014).

[24] L. Mandel and E. Wolf, Optical Coherence and Quantum Optics (Cambridge University Press, Cambridge, England, 1995).

[25] L. Novotny and B. Hecht, Principles of Nano-optics (Cambridge University Press, Cambridge, England, 2006).

[26] C. Sauvan, J. P. Hugonin, R. Carminati, and P. Lalanne, Modal Representation of Spatial Coherence in Dissipative, and Resonant Photonic Systems, Phys. Rev. A 89, 043825 (2014).

[27] U. Fano, Effects of Configuration Interaction on Intensities, and Phase Shifts, Phys. Rev. 124, 1866 (1961).

[28] https://www.lp2n.institutoptique.fr/Membres-Services/ Responsables-d-equipe/LALANNE-Philippe.

[29] A. F. Koenderink, On the Use of Purcell Factors for Plasmon Antennas, Opt. Lett. 35, 4208 (2010).

[30] M. O. Scully and M.S. Zubairy, Quantum Optics (Cambridge University Press, Cambridge, England, 1997).

[31] C. F. Bohren and D. R. Huffman, Absorption and Scattering of Light by Small Particles (Wiley, New York, 1983). 